\title{
Development and validation of radiologic scores for guiding individualized induction chemotherapy in T3N1M0 nasopharyngeal carcinoma
}

\author{
Shan-Shan Yang ${ }^{1} \cdot{\text { Yi-Shan } \mathrm{Wu}^{2} \cdot \text { Ya-Jun Pang }}^{3} \cdot$ Su-Ming Xiao ${ }^{1} \cdot$ Bao-Yu Zhang ${ }^{1} \cdot$ Zhi-Qiao Liu $^{1} \cdot$ En-Ni Chen ${ }^{1}$. \\ Xu Zhang ${ }^{4} \cdot$ Pu-Yun OuYang ${ }^{1} \cdot$ Fang-Yun Xie $^{1}$ (i)
}

Received: 8 June 2021 / Revised: 2 November 2021 / Accepted: 5 November 2022 / Published online: 6 January 2022

(c) The Author(s) 2022

\begin{abstract}
Objectives We aimed to develop and validate radiologic scores from $\left[{ }^{18} \mathrm{~F}\right] \mathrm{FDG}$ PET/CT and MRI to guide individualized induction chemotherapy (IC) for patients with T3N1M0 nasopharyngeal carcinoma (NPC).

Methods A total of 542 T3N1M0 patients who underwent pretreatment $\left[{ }^{18} \mathrm{~F}\right] \mathrm{FDG}$ PET/CT and MRI were enrolled in the training cohort. A total of 174 patients underwent biopsy of one or more cervical lymph nodes. Failure-free survival (FFS) was the primary endpoint. The radiologic score, which was calculated according to the number of risk factors from the multivariate model, was used for risk stratification. The survival difference of patients undergoing concurrent chemoradiotherapy (CCRT) with or without IC was then compared in risk-stratified subgroups. Another cohort from our prospective clinical trial $(N=353$, NCT03003182) was applied for validation.

Results The sensitivity of $\left[{ }^{18}\right.$ F]FDG PET/CT was better than that of MRI $(97.7 \%$ vs. $87.1 \%, p<0.001)$ for diagnosing histologically proven metastatic cervical lymph nodes. Radiologic lymph node characteristics were independent risk factors for FFS (all $p<0.05)$. High-risk patients $(n=329)$ stratified by radiologic score benefited from IC (5-year FFS: IC + CCRT $83.5 \%$ vs. CCRT $70.5 \% ; p=0.0044)$, while low-risk patients $(n=213)$ did not. These results were verified again in the validation cohort.

Conclusions T3N1M0 patients were accurately staged by both $\left[{ }^{18}\right.$ F]FDG PET/CT and MRI. The radiologic score can correctly identify high-risk patients who can gain additional survival benefit from IC and it can be used to guide individualized treatment of T3N1M0 NPC.
\end{abstract}

Shan-Shan Yang and Yi-Shan Wu contributed equally to this work and shared the first authorship.

Senior authors Pu-Yun OuYang and Fang-Yun Xie contributed equally.

Fang-Yun Xie

xiefy@sysucc.org.cn

1 Department of Radiation Oncology, Sun Yat-Sen University Cancer Center, State Key Laboratory of Oncology in South China, Collaborative Innovation Center for Cancer Medicine, Guangdong Key Laboratory of Nasopharyngeal Carcinoma Diagnosis and Therapy, No. 651 Dongfeng Road East, Guangzhou 510060, China

2 Department of Nasopharyngeal Carcinoma, Sun Yat-Sen University Cancer Center, State Key Laboratory of Oncology in South China, Collaborative Innovation Center for Cancer Medicine, Guangdong Key Laboratory of Nasopharyngeal Carcinoma Diagnosis and Therapy, No. 651 Dongfeng Road East, Guangzhou 510060, China
3 Cancer Center, Affiliated Hospital of Guangdong Medical University, Renmin Avenue, Xiashan District, Zhanjiang, China

4 Department of Nuclear Medicine, Sun Yat-Sen University Cancer Center, State Key Laboratory of Oncology in South China, Collaborative Innovation Center for Cancer Medicine, Guangdong Key Laboratory of Nasopharyngeal Carcinoma Diagnosis and Therapy, No. 651 Dongfeng Road East, Guangzhou 510060, China 
Key Points

- $\left[{ }^{18} F\right] F D G$ PET/CT was more accurate than MRI in diagnosing histologically proven cervical lymph nodes.

- Radiologic lymph node characteristics were reliable independent risk factors for FFS in T3N1MO nasopharyngeal carcinoma patients.

- High-risk patients identified by the radiologic score based on $\left[{ }^{18} \mathrm{~F}\right] F D G$ PET/CT and MRI could benefit from the addition of induction chemotherapy.

Keywords Nasopharyngeal carcinoma $\cdot\left[{ }^{18} \mathrm{~F}\right] \mathrm{FDG}$ PET/CT $\cdot$ MRI $\cdot$ Induction chemotherapy

\section{Abbreviations}

$\left[{ }^{18}\right.$ F]FDG PET/CT 2-Deoxy-2- $\left[{ }^{18}\right.$ F] fluoro-D-glucose positron emission tomography/computed tomography

AJCC/UICC

AUC

CCRT

CI

CTV

DMFS

EBV

FFS

FN

FP

GP

HR

IC

$\mathrm{K}-\mathrm{M}$

LDH

MRI

$\mathrm{NCCN}$

NPC

NPV

OS

OSEM

PF

PPV

rENE

ROC

RRFS

SUVmax-N

SUVmax-T

TN

TP

TP

TPF
American Joint Committee on Cancer/Union for International Cancer

Control

Area under the curve

Concurrent chemoradiotherapy

Confidence interval

Clinical target volume

Distant metastasis-free survival

Epstein-Barr virus

Failure-free survival

False-negative

False-positive

Gemcitabine/cisplatin

Hazard ratio

Induction chemotherapy

Kaplan-Meier

Serum lactate dehydrogenase

Magnetic resonance imaging

National Comprehensive Cancer

Network

Nasopharyngeal carcinoma

Negative predictive value

Overall survival

Ordered subset expectation

maximization

Cisplatin/5-fluorouracil

Positive predictive value

Radiologic extranodal extension

Receiver operating characteristic

Regional relapse-free survival

The maximal standardized uptake value of lymph node

The maximal standardized uptake value of the primary tumor

True negative

Docetaxel/cisplatin

True positive

Docetaxel/cisplatin/5-fluorouracil

\section{Introduction}

In 2020, 133,354 new cases of nasopharyngeal carcinoma were reported, accounting for $0.7 \%$ of all cancers in the world, but over $70 \%$ of patients were from Asia, with an age standardized rate (world) of 3.0 per 100,000 in China $[1,2]$. Unfortunately, over $75 \%$ of patients are diagnosed with locoregionally advanced disease at presentation [3]. Despite advances in techniques, nearly $30 \%$ of patients experience treatment failure, especially distant metastasis [4]. Phase III randomized controlled trials have proven that induction chemotherapy added to concurrent chemoradiotherapy can significantly decrease the risk of distant metastasis and improve the survival of patients with locoregionally advanced nasopharyngeal carcinoma [5-7]. This treatment mode is thus the category $2 \mathrm{~A}$ recommendation for these patients by National Comprehensive Cancer Network (NCCN) guidelines [8]. However, notably, these randomized trials did not enroll any patients staged with T3-4N0M0 or T3N1M0 at all. A retrospective study reported that patients with T3N0-1 do not benefit from induction chemotherapy [9], while male T3N1 patients with Epstein-Barr virus (EBV) DNA higher than 2000 copies/mL were the only target population for induction followed by concurrent chemoradiotherapy, as suggested by another study [10]. Therefore, the treatment modality of T3N1 nasopharyngeal carcinoma is still controversial. Although EBV DNA has been reported to have prognostic value, its extensive application is difficult in real-world practice due to the lack of recognized cutoff values and unified test standards. 2-Deoxy-2- $\left[{ }^{18} \mathrm{~F}\right]$ fluoro-D-glucose $\left(\left[{ }^{18} \mathrm{~F}\right] \mathrm{FDG}\right)$ positron emission tomography/ computed tomography (PET/CT) and magnetic resonance imaging (MRI) have been widely applied for the diagnosis and staging of nasopharyngeal carcinoma [11]. Given the widespread use of $\left[{ }^{18} \mathrm{~F}\right] \mathrm{FDG}$ PET/CT and MRI, the radiologic characteristics of the primary tumor and metastatic lymph nodes may prove useful for selecting individualized treatment.

The maximal standardized uptake value (SUVmax) of $\left[{ }^{18} \mathrm{~F}\right] \mathrm{FDG} \mathrm{PET} / \mathrm{CT}$, related to metabolic activity, has prognostic implications and is used for risk stratification [12, 13]. The SUVmax of lymph nodes (SUVmax-N) and the lymph node-to-primary tumor SUVmax ratio are potential 
prognostic factors in nasopharyngeal carcinoma patients $[12,14]$. However, the prognostic value of the SUVmax of the primary tumor (SUVmax-T) is in dispute [15]. Previous studies showed that ungraded radiologic extranodal extension determined by MRI had no prognostic significance in nasopharyngeal carcinoma [16, 17]. After grading the radiologic extranodal extension, the sensitivity of diagnosing pathologic extranodal extension improved in head and neck cancer [18], and the consistency of determining radiologic extranodal extension also increased as extranodal extension grades increased. Recent studies demonstrated that high-grade radiologic extranodal extension with adjacent structure invasion significantly predicted a poor survival outcome [19-22]. However, the above studies did not eliminate the interference of confounding factors such as $\mathrm{T}$ stage, cervical lymph node level, laterality, and necrosis status. Thus, the reported prognostic value of radiologic characteristics in prior studies needs re-evaluation.

Additionally, accurate diagnosis of T3N1M0 patients is another key point. $\left[{ }^{18} \mathrm{~F}\right] \mathrm{FDG}$ PET/CT has advantages in detecting metastatic cervical lymph nodes and distant metastasis over MRI, but it is inferior in determining local tumor invasion and retropharyngeal nodal metastasis [23, 24]. This conclusion was based on the judgment of metastatic lymph nodes by clinical follow-up instead of pathologic confirmation. Herein, we included patients who underwent both MRI and $\left[{ }^{18} \mathrm{~F}\right] \mathrm{FDG}$ PET/CT examination before treatment. More importantly, one or more cervical lymph nodes of certain patients were histologically confirmed, so the performance of $\left[{ }^{18} \mathrm{~F}\right] \mathrm{FDG}$ PET/CT and MRI in diagnosing the specific lymph nodes provided firm evidence for precisely identifying the subgroup of T3N1M0. Subsequently, we accurately developed and validated the radiologic score of the lymph node characteristics to identify high-risk patients who can gain an additional survival benefit from induction chemotherapy and finally suggested an individualized treatment mode for these patients.

\section{Materials and methods}

\section{Patients}

A training cohort of 542 patients staged with T3N1M0 nasopharyngeal carcinoma by pretreatment MRI and $\left[{ }^{18} \mathrm{~F}\right] \mathrm{FDG}$ PET/CT was obtained from May 2009 to May 2017 at Sun Yat-sen University Cancer Center. The eligibility criteria for selecting participants were as follows: (1) newly diagnosed nasopharyngeal carcinoma restaged as T3N1M0 in accordance with the 8th edition American Joint Committee on Cancer/Union for International Cancer Control (AJCC/ UICC) staging system [25]; (2) receipt of pretreatment MRI and $\left[{ }^{18} \mathrm{~F}\right] \mathrm{FDG}$ PET/CT tests simultaneously; (3) receipt of concurrent chemoradiotherapy or induction followed by concurrent chemoradiotherapy; and (4) receipt of intensitymodulated radiotherapy. The patient flow chart is presented in Fig. 1.

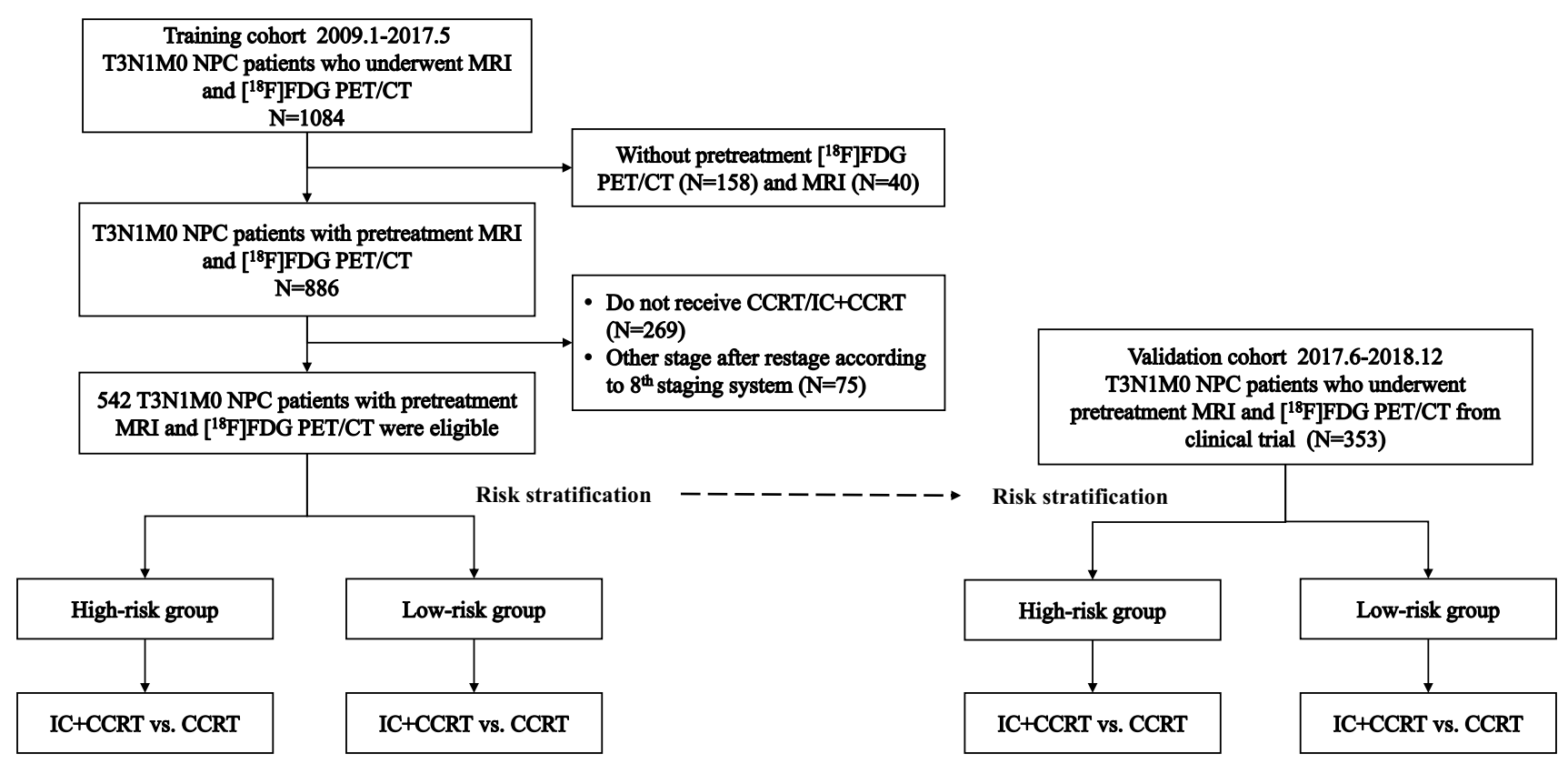

Fig. 1 The flowchart of the included patients. CCRT, concurrent chemoradiotherapy; IC, induction chemotherapy; MRI, magnetic resonance imaging; NPC, nasopharyngeal carcinoma; $\left[{ }^{18} \mathrm{~F}\right] \mathrm{FDG}$ PET/
CT, 2-deoxy-2-[ ${ }^{18}$ F]fluoro-D-glucose positron emission tomography/ computed tomography 
A validation cohort containing 345 patients was obtained from our prospective clinical trial (ClinicalTrials.gov Identifier: NCT03003182) between June 2017 and September 2018.

This study was approved by the Institutional Ethical Review Board (No. B2021-059-01), and informed consent was waived for the part of retrospective analysis. Patients in the validation cohort were derived from a prospective observational study, and informed consent regarding a second analysis of their data was obtained from all of the patients.

\section{Image analysis}

All patients received whole-body $\left[{ }^{18} \mathrm{~F}\right] \mathrm{FDG}$ PET/CT and MRI examinations of the head and neck. The detailed MRI and $\left[{ }^{18} \mathrm{~F}\right]$ FDG PET/CT protocols are shown in the Supplementary Methods. All $\left[{ }^{18} \mathrm{~F}\right]$ FDG PET/CT images were evaluated by a researcher (SSY, 5 years of experience in treating nasopharyngeal carcinoma) with reference to the issued report and then checked by an expert nuclear medicine physician (X.Z., more than 20 years of experience). All MR images were assessed by a radiation oncologist (P.Y.O.Y.) with 10 years of experience and reviewed again by an expert radiation oncologist (F.Y.X.) with over 30 years of experience in treating nasopharyngeal carcinoma. Inconsistencies were discussed with a radiologist (Y.H.) who had interpreted head and neck MR images of over 500 patients per month for over 5 years.

The diagnostic criteria for the metastatic lymph nodes, radiologic extranodal extension, and nodal necrosis were the same as those in previous studies [19, 26, 27] and are detailed in the Supplementary Methods. SUVmax was defined as the highest decay-corrected activity concentration per injected dose per body weight. The treatment and follow-up are shown in the Supplementary Methods.

\section{Statistical analysis}

The primary endpoint was failure-free survival (FFS), which was defined as the time from diagnosis to failure (locoregional recurrence or distant metastasis) or death. The secondary endpoints were overall survival (OS, from diagnosis to death from any cause), regional relapse-free survival (RRFS, from diagnosis to regional recurrence or death), and distant metastasis-free survival (DMFS, from diagnosis to distant metastasis or death).

The sensitivity and specificity of $\left[{ }^{18} \mathrm{~F}\right] \mathrm{FDG}$ PET/CT and MRI were compared using McNemar's paired-sample test, and confidence intervals for proportions were calculated according to the efficient-score method described by Robert Newcombe [28]. Time-dependent receiver operating characteristic (ROC) curve analysis was applied to determine the cutoff values of the continuous variables using the "survival ROC" package in R. The survival curves were compared by the log-rank test. Univariate and multivariate analyses were performed by Cox regression. Statistical analysis was conducted using SPSS 26.0 and R software (version 4.0.1, http://www.r-project.org/). A two-sided $p<0.05$ was deemed statistically significant.

\section{Results}

\section{Patient characteristics}

After screening, 542 and 353 eligible patients were enrolled in the training cohort and the validation cohort, respectively. In the training cohort, the median age was 44 years, ranging from 16 to 73 years. The cutoff value of SUVmax-N was 9.3 for FFS (area under the curve [AUC]: 0.616, $p=0.001$; Supplementary Fig. 2). The pretreatment EBV DNA cutoff point (2000 copies/mL) was determined according to previous studies and ROC curves. With a median follow-up time of 61 (range, 2-118) months for the 542 patients, 103 (19.0\%) patients had treatment failure, and $38(7.0 \%)$ patients suffered from regional recurrence. In addition, 7.4\% (40/542) of patients had distant metastasis, while 6.6\% (36/542) of patients died at the last follow-up. The 5-year FFS, RRFS, DMFS, and OS rates were $82.2 \%, 93.7 \%, 92.6 \%$, and $94.5 \%$, respectively. The baseline characteristics of the enrolled patients are shown in Table 1.

\section{$\left[{ }^{18} \mathrm{~F}\right]$ FDG PET/CT versus MRI}

In the whole cohort, 174 patients underwent cervical lymph node fine-needle aspiration biopsy guided by ultrasonography. Among the 224 biopsied lymph nodes of 174 patients, 132 and 92 lymph nodes were pathologically confirmed positive and negative, respectively. $\left[{ }^{18} \mathrm{~F}\right] \mathrm{FDG} \mathrm{PET} / \mathrm{CT}$ correctly diagnosed 129 positive lymph nodes and 74 negative lymph nodes, whereas MRI correctly found 115 positive lymph nodes and 59 negative lymph nodes. The sensitivity of $\left[{ }^{18} \mathrm{~F}\right]$ FDG PET/CT $(97.7 \%)$ was higher than that of MRI $(87.1 \%)$ for detecting cervical lymph nodes $(p<0.001)$. The specificity, positive predictive value, and negative predictive value of $\left[{ }^{18} \mathrm{~F}\right] \mathrm{FDG}$ PET/CT and MRI were $80.4 \%$ vs. $64.1 \%, 87.8 \%$ vs. $77.7 \%$, and $96.1 \%$ vs. $77.6 \%$, respectively (see Table 2). As a result, 39 of 174 patients with lymph node biopsy showed an inconsistent $\mathrm{N}$ stage between $\left[{ }^{18} \mathrm{~F}\right]$ FDG PET/CT and MRI. In the whole training cohort, 472 patients were consistently staged as T3 by $\left[{ }^{18} \mathrm{~F}\right] \mathrm{FDG}$ PET/ CT or MRI; however, 20, 77, and 25 patients were possibly classified as N0, N2, or N3 by mistake if staged by MRI alone. 
Table 1 Baseline characteristics in the training and validation cohorts

\begin{tabular}{|c|c|c|}
\hline \multirow[t]{2}{*}{ Characteristics } & \multicolumn{2}{|c|}{ Number of patients $(\%)$} \\
\hline & $\begin{array}{l}\text { Training cohort } \\
(n=542)\end{array}$ & $\begin{array}{l}\text { Validation cohort } \\
(n=353)\end{array}$ \\
\hline Median age (range) & $44(16-73)$ & $48(13-69)$ \\
\hline$<52$ & $399(73.6)$ & $230(65.2)$ \\
\hline$\geq 52$ & $143(26.4)$ & $123(34.8)$ \\
\hline \multicolumn{3}{|l|}{ Sex } \\
\hline Male & $381(70.3)$ & $247(70.0)$ \\
\hline Female & $161(29.7)$ & $106(30.0)$ \\
\hline \multicolumn{3}{|l|}{ rENE } \\
\hline Grade 0 & $260(48.0)$ & $204(57.8)$ \\
\hline Grade 1 & 107 (19.7) & $62(17.6)$ \\
\hline Grade 2 & $111(20.5)$ & $58(16.4)$ \\
\hline Grade 3 & $64(11.8)$ & $29(8.2)$ \\
\hline \multicolumn{3}{|l|}{ Nodal necrosis } \\
\hline Yes & $93(17.2)$ & $54(15.3)$ \\
\hline No & $449(82.8)$ & 299 (84.7) \\
\hline \multicolumn{3}{|l|}{ SUVmax-N } \\
\hline$<9.3$ & $239(44.1)$ & $229(64.9)$ \\
\hline$\geq 9.3$ & $303(55.9)$ & $124(35.1)$ \\
\hline \multicolumn{3}{|l|}{ SUVmax-T } \\
\hline$<16.3$ & $455(83.9)$ & $279(79.0)$ \\
\hline$\geq 16.3$ & $87(16.1)$ & $74(21.0)$ \\
\hline Minimal axial diameter, median (range) $\mathrm{cm}$ & $1.2(0.3-4.1)$ & $1.1(0.4-3.0)$ \\
\hline Maximal axial diameter, median (range) $\mathrm{cm}$ & $1.7(0.5-5.3)$ & $1.1(0.5-4.9)$ \\
\hline \multicolumn{3}{|l|}{ Lymph node } \\
\hline Retropharyngeal lymph node & $92(17.0)$ & $92(26.1)$ \\
\hline Cervical lymph node & $450(83.0)$ & $261(73.9)$ \\
\hline \multicolumn{3}{|l|}{ EBV DNA (copy/mL) } \\
\hline$<2000$ & $336(62.0)$ & 257 (72.8) \\
\hline$\geq 2000$ & $206(38.0)$ & $96(27.2)$ \\
\hline \multicolumn{3}{|l|}{ Hemoglobin (g/L) } \\
\hline$<120$ & $18(3.3)$ & $14(4.0)$ \\
\hline$\geq 120$ & $524(96.7)$ & $339(96.0)$ \\
\hline \multicolumn{3}{|l|}{ LDH (U/L) } \\
\hline$<250$ & $520(95.9)$ & $344(97.5)$ \\
\hline$\geq 250$ & $22(4.1)$ & $9(2.5)$ \\
\hline \multicolumn{3}{|l|}{ Albumin $(\mathrm{g} / \mathrm{L})$} \\
\hline$<40$ & $29(5.4)$ & $8(2.3)$ \\
\hline$\geq 40$ & $513(94.7)$ & $345(97.7)$ \\
\hline \multicolumn{3}{|l|}{ Treatment } \\
\hline $\mathrm{IC}+\mathrm{CCRT}$ & $226(41.7)$ & $131(37.1)$ \\
\hline CCRT & $316(58.3)$ & $222(62.9)$ \\
\hline \multicolumn{3}{|l|}{ IC regimen } \\
\hline $\mathrm{TPF}$ & $64(11.8)$ & $39(11.0)$ \\
\hline $\mathrm{TP}$ & $82(15.1)$ & $53(15.0)$ \\
\hline $\mathrm{PF}$ & $69(12.7)$ & $28(7.9)$ \\
\hline GP & $11(2.0)$ & $11(3.1)$ \\
\hline
\end{tabular}

$C C R T$, concurrent chemoradiotherapy; $E B V$, Epstein-Barr virus; $G P$, gemcitabine/cisplatin; $I C$, induction chemotherapy; $L D H$, serum lactate dehydrogenase; $P F$, cisplatin/5-fluorouracil; $r E N E$, radiologic extranodal extension; SUVmax-N, the maximal standardized uptake value of lymph node; SUVmax-T, the maximal standardized uptake value of primary tumor; $T P$, docetaxel/cisplatin; $T P F$, docetaxel/cisplatin/5-fluorouracil 
Table 2 Results of $\left[{ }^{18} \mathrm{~F}\right] \mathrm{FDG}$ PET/CT and MRI in detecting cervical lymph nodes confirmed by histopathology in 174 patients

\begin{tabular}{lllr}
\hline & {$\left[{ }^{18}\right.$ F $]$ FDG PET/CT } & MRI & $p$ \\
\hline TP & 129 & 115 & \\
TN & 74 & 59 & \\
FP & 18 & 33 & \\
FN & 3 & 17 & \\
Sensitivity (95\% & $97.7(93.0-99.4)$ & $87.1(79.9-92.1)$ & $<0.001$ \\
$\quad$ CI) & & & \\
Specificity (95\% & $80.4(70.6-87.7)$ & $64.1(53.4-73.7)$ & $<0.001$ \\
CI) & & & 0.022 \\
PPV (95\% CI) & $87.8(81.1-92.4)$ & $77.7(70.0-84.0)$ & \\
NPV (95\% CI) & $96.1(88.3-99.0)$ & $77.6(66.4-86.1)$ & $<0.001$ \\
\hline
\end{tabular}

$C I$, confidence interval; $F P$, false-positive; $F N$, false-negative; $N P V$, negative predictive value; $M R I$, magnetic resonance imaging; $\left[{ }^{18} \mathrm{~F}\right]$ $F D G$ PET/CT, 2-deoxy-2- $\left[{ }^{18}\right.$ F $]$ fluoro-D-glucose positron emission tomography/computed tomography; $P P V$, positive predictive value; $T P$, true positive; $T N$, true negative

\section{Radiologic characteristics and survival outcomes}

Of 542 patients, 93 (17.2\%) patients had nodal necrosis. A total of $303(55.9 \%)$ patients were assigned to the higher SUVmax-N group ( $\geq 9.3)$, and $87(16.1 \%)$ patients had an SUVmax-T higher than 16.3. The median maximal axial diameter was 1.7 (range, $0.5-5.3$ ) $\mathrm{cm}$. The proportion of patients with grade 0 , grade 1 , grade 2 , and grade 3 radiologic extranodal extension was $48.0 \%$ (260/542), $19.7 \%$ (107/542), 20.5\% (111/542), and 11.8\% (64/542), respectively, in the training cohort.

The analysis showed that patients with grade 3 radiologic extranodal extension had significantly lower FFS than those with grades 0,1 , and 2 radiologic extranodal extension $(p<0.001, p<0.001$, and $p=0.003)$. The survival curve is shown in Supplementary Fig. 3. As presented in Table 3, multivariate analysis demonstrated that SUVmax-N higher than 9.3 , nodal necrosis, and grade 3 radiologic extranodal extension were independent factors of a poor prognosis for FFS ( $p=0.035, p<0.001$, and $p=0.001$, respectively). For graded radiologic extranodal extension, only grade 3 , but not grade $0-2$ radiologic extranodal extension, predicted an inferior FFS (hazard ratio [HR]: 2.703, 95\% CI: 1.547-4.724, $p=0.001$; Table 3 ).

Similarly, the above lymph node characteristics were also independent factors for DMFS, while grade 3 radiologic extranodal extension was the only significant independent factor for RRFS (Supplementary Table 1).

\section{Radiologic score and risk stratification}

Prognostic factors obtained from the multivariate analysis were used for risk stratification. One risk factor scored
Table 3 Univariate and multivariable analysis of FFS in the training cohort

\begin{tabular}{|c|c|c|c|c|}
\hline \multirow[t]{2}{*}{ Variable } & \multicolumn{2}{|l|}{ Univariate analysis } & \multicolumn{2}{|l|}{ Multivariable analysis } \\
\hline & HR $(95 \% \mathrm{CI})$ & $p$ & $\mathrm{HR}(95 \% \mathrm{CI})$ & $p$ \\
\hline Age $(\geq 52$ vs. $<52)$ & $1.145(0.745-1.761)$ & 0.537 & & \\
\hline Sex (male vs. female) & $0.879(0.578-1.336)$ & 0.546 & & \\
\hline \multicolumn{5}{|l|}{ rENE } \\
\hline Grade 0 & Reference & & Reference & \\
\hline Grade 1 & $1.335(0.762-2.341)$ & 0.313 & $1.097(0.617-1.952)$ & 0.752 \\
\hline Grade 2 & 1.719 (1.019-2.899) & 0.042 & $1.233(0.705-2.157)$ & 0.463 \\
\hline Grade 3 & $3.983(2.373-6.685)$ & $<0.001$ & $2.703(1.547-4.724)$ & 0.001 \\
\hline Nodal necrosis (yes vs. no) & $2.544(1.664-3.890)$ & $<0.001$ & $1.897(1.220-2.949)$ & $<0.001$ \\
\hline SUVmax-N $(\geq 9.3$ vs. $<9.3)$ & $2.240(1.444-3.475)$ & $<0.001$ & $1.672(1.037-2.697)$ & 0.035 \\
\hline SUVmax-T $(\geq 16.3$ vs. $<16.3)$ & $1.145(0.745-1.761)$ & 0.456 & & \\
\hline Minimal axial diameter & $1.562(1.201-2.031)$ & 0.001 & & \\
\hline Maximal axial diameter & $1.353(1.095-1.671)$ & 0.005 & & \\
\hline $\begin{array}{l}\text { Lymph node (cervical lymph node } \\
\text { vs. retropharyngeal lymph node) }\end{array}$ & $2.139(1.142-4.007)$ & 0.018 & & \\
\hline EBV DNA ( $\geq 2000$ vs. $<2000$ ) & $1.340(0.907-1.980)$ & 0.141 & & \\
\hline Hemoglobin $(\geq 120$ vs. $<120)$ & $2.230(0.533-9.328)$ & 0.272 & & \\
\hline LDH ( $\geq 250$ vs. $<250)$ & $0.748(0.237-2.360)$ & 0.620 & & \\
\hline Albumin $(\geq 40$ vs. $<40)$ & $0.535(0.270-1.063)$ & 0.074 & & \\
\hline Treatment $(\mathrm{IC}+\mathrm{CCRT}$ vs. CCRT $)$ & $0.728(0.485-1.093)$ & 0.126 & & \\
\hline
\end{tabular}

$C I$, confidence interval; $C C R T$, concurrent chemoradiotherapy; $E B V$, Epstein-Barr virus; $F F S$, failure-free survival; $H R$, hazard ratio; $I C$, induction chemotherapy; $L D H$, serum lactate dehydrogenase; $r E N E$, radiologic extranodal extension; $S U V \max -N$, the maximal standardized uptake value of lymph node; $S U V \max -T$, the maximal standardized uptake value of primary tumor 
a

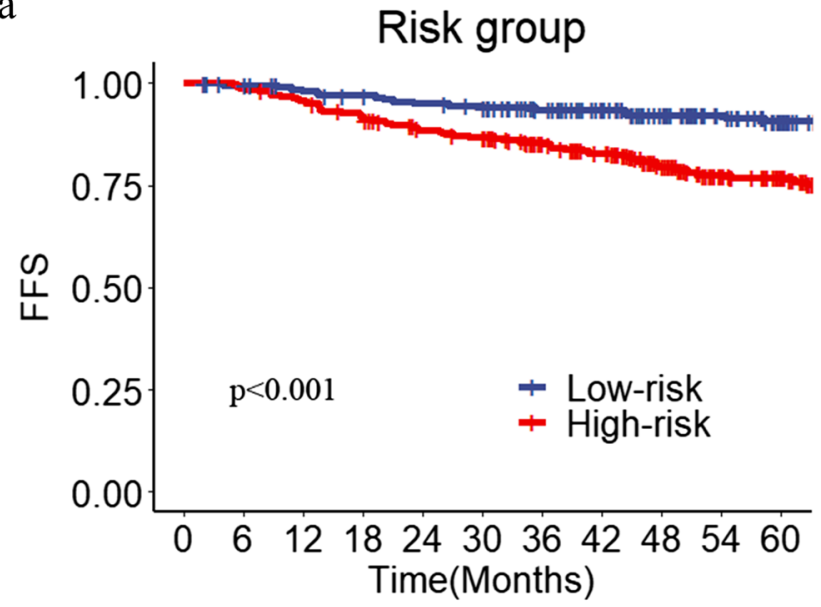

Number at risk

Low-risk 213208200196191188173161141120102

High-risk 329325314298282275253233200164148

$\mathrm{C}$

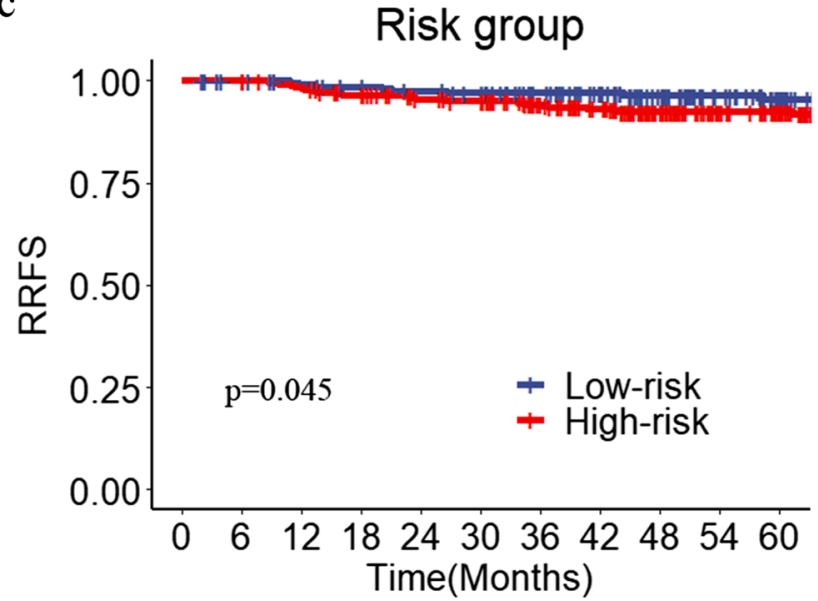

Number at risk

Low-risk 213208201197193189175163144122105

High-risk 329329322310297292270250215179162

Fig. 2 Survival curves of high- and low-risk groups stratified by radiologic score in FFS (a), DMFS (b), RRFS (c), and OS (d) in the training cohort. High-risk group: radiologic score $>0$, low-risk group:

1 point, and patients were thus scaled from 0 to 3 points according to the number of risk factors. The survival curve revealed that patients with higher radiologic scores had lower survival rates ( $p<0.001$; Supplementary Fig. 4). Therefore, patients were stratified into a high-risk group (radiologic score $>0, n=329$ ) and a low-risk group (radiologic score $=0, n=213$ ) by their radiologic score. The baseline characteristics of participants in both risk groups are summarized in Supplementary Table 2. As shown in Fig. 2, the 5-year FFS, DMFS, RRFS, and OS rates were 90.7\% vs. $77.0 \%, 98.7 \%$ vs. $88.8 \%, 95.5 \%$ vs. $92.5 \%$, and $97.3 \%$ b

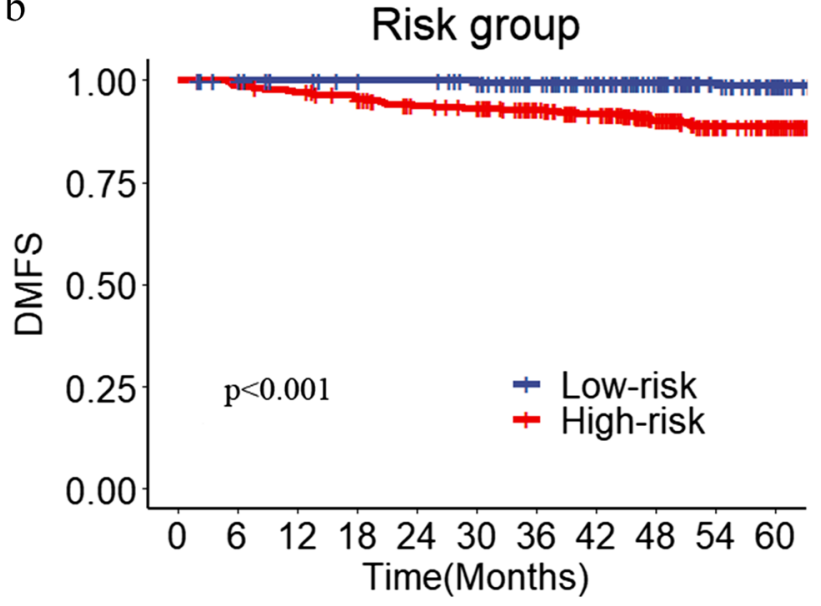

Number at risk

Low-risk 213209204201200196181169150126109

High-risk 329325319309295290270250217179160

d

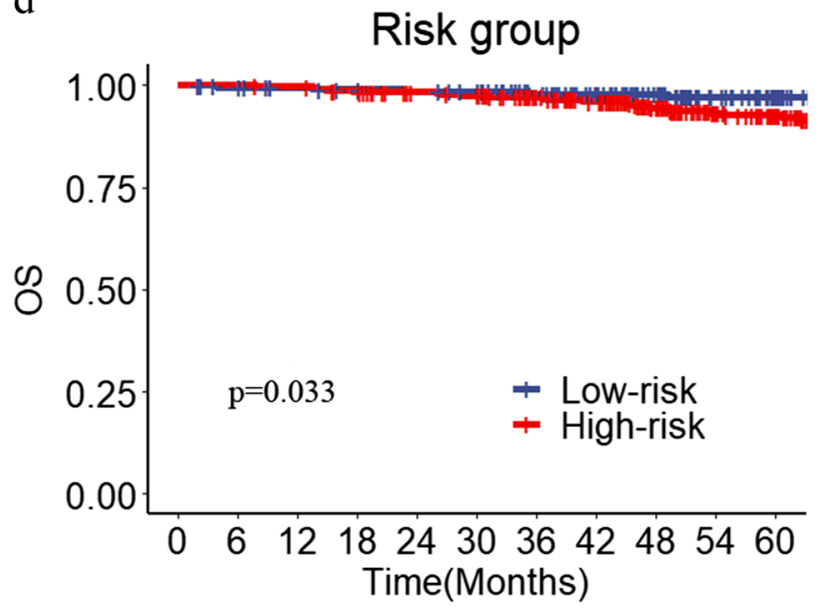

Number at risk

Low-risk 213208203200198195181169150126110

High-risk 329329327321310306286267232193173

radiologic score $=0$. DMFS, distant metastasis-free survival; FFS, failure-free survival; OS, overall survival; RRFS, regional relapsefree survival

vs. $92.8 \%$ for patients in the low-risk and high-risk groups, respectively (all $p<0.05$ ).

\section{Benefit of induction chemotherapy}

In the whole training cohort, FFS was not significantly different for patients with or without induction chemotherapy before concurrent chemoradiotherapy (HR: $0.72,95 \%$ CI: $0.49-1.09, p=0.12$ by univariate analysis; Fig. 3a). However, in the high-risk group, patients who received induction followed by concurrent 

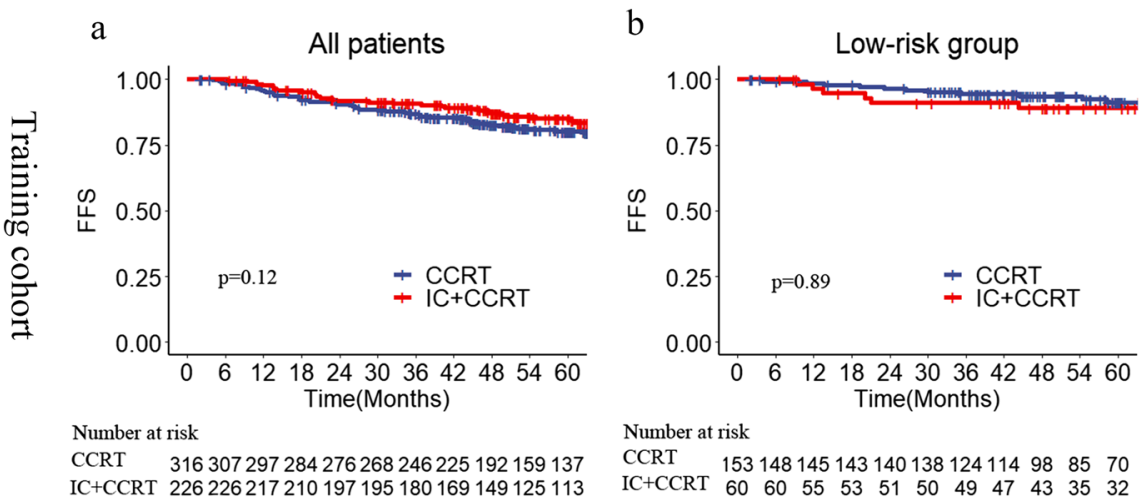

Number at risk

CCRT $15314814514314013812411498 \quad 85 \quad 70$ $\begin{array}{llllllllllll}\text { IC+CCRT } & 60 & 60 & 55 & 53 & 51 & 50 & 49 & 47 & 43 & 35 & 32\end{array}$

e

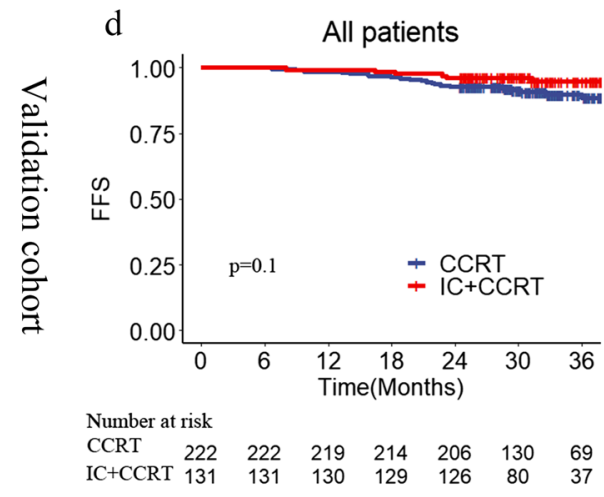

c

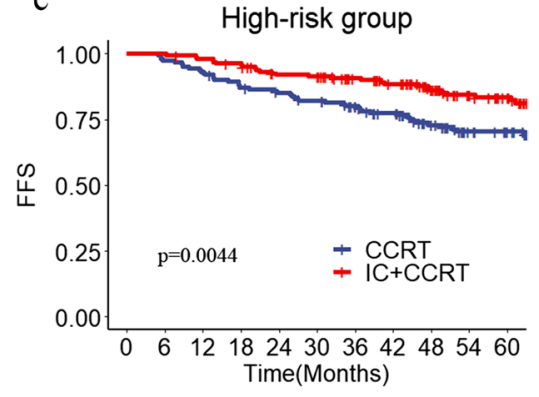

Number at risk

CCRT $16315915214113613012211194 \quad 74 \quad 67$ IC+CCRT 1661661621571461451311221069081

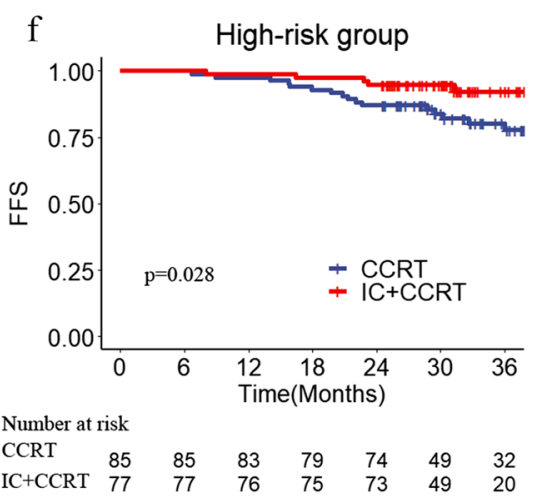

Fig. 3 Kaplan-Meier FFS curves of IC + CCRT and CCRT alone in the training cohort (whole cohort (a), low-risk group (b), high-risk group (c)) and validation cohort (whole cohort (d), low-risk group

chemoradiotherapy had better FFS than patients who received concurrent chemoradiotherapy alone (5-year FFS: $83.5 \%$ vs. $70.5 \%, p=0.0044$; Fig. 3c). Additionally, multivariate Cox regression analysis indicated that induction chemotherapy plus concurrent chemoradiotherapy was still an independent prognostic factor for FFS (HR: $0.48,95 \%$ CI: $0.305-0.755, p=0.002$; Table 4 ) in the (e), high-risk group (f)). CCRT, concurrent chemoradiotherapy; FFS, failure-free survival; IC, induction chemotherapy

high-risk group. In the low-risk group, similar survival outcomes were observed between induction chemotherapy followed by concurrent chemoradiotherapy and concurrent chemoradiotherapy alone (5-year FFS: $91.3 \%$ vs. $89.1 \%, p=0.89$; Fig. 3 ).

The results for DMFS and RRFS are shown in Supplementary Fig. 5, Supplementary Table 3, and Table 4.
Table 4 Results of multivariate analysis for the high-risk group in the training cohort

\begin{tabular}{lllr}
\hline Endpoints & Variables & HR $(95 \%$ CI $)$ & $p$ \\
\hline FFS & rENE (grade 3 vs. grades 0-2) & $2.508(1.557-4.040)$ & $<0.001$ \\
& Nodal necrosis (yes vs. no) & $1.780(1.133-2.795)$ & 0.012 \\
& Treatment (IC + CCRT vs. CCRT) & $0.480(0.305-0.755)$ & 0.002 \\
DMFS & rENE (grade 3 vs. grades 0-2) & $2.046(1.004-4.173)$ & 0.045 \\
& Nodal necrosis (yes vs. no) & $2.085(1.081-4.021)$ & 0.028 \\
& Treatment (IC + CCRT vs. CCRT) & $0.460(0.234-0.904)$ & 0.024 \\
RRFS & Nodal necrosis (yes vs. no) & $2.461(1.115-5.429)$ & 0.026 \\
& Treatment (IC + CCRT vs. CCRT) & $0.409(0.186-0.902)$ & 0.027 \\
OS & Age ( $\geq 52$ vs. $<52)$ & $2.546(1.213-5.346)$ & 0.014 \\
& Albumin $(\geq 40$ vs. <40) & $0.328(0.125-0.863)$ & 0.024 \\
\hline
\end{tabular}

$C I$, confidence interval; $C C R T$, concurrent chemoradiotherapy; DMFS, distant metastasis-free survival; $F F S$, failure-free survival; $H R$, hazard ratio; $I C$, induction chemotherapy; $O S$, overall survival; $r E N E$, radiologic extranodal extension; RRFS, regional relapse-free survival 


\section{Validation}

As shown in Table 1, the median age was 48 years (range, 13-69) in the validation cohort. With a median follow-up time of 32 (range, 14-44) months for these 353 patients, 27 developed treatment failure. The 3-year FFS and RRFS were $90.8 \%$ and $93.7 \%$, respectively. Patients with nodal necrosis accounted for $15.3 \%$, and 124 patients had a higher SUVmax-N ( $\geq 9.3$ ). Among 353 patients, 204 (57.8\%), 62 (17.6\%), $58(16.4 \%)$, and $29(8.2 \%)$ patients had grade 0 , grade 1 , grade 2 , and grade 3 radiologic extranodal extension, respectively. Grade 3 radiologic extranodal extension, SUVmax-N ( $\geq 9.3$ ), and nodal necrosis were confirmed as significant factors of a poor prognosis for FFS in the validation group (all $p<0.05$; Supplementary Fig. 6).

The prospective validation set was stratified into a highrisk group $(n=162)$ and a low-risk group $(n=191)$ according to the radiologic score identified in the training set. The 3-year FFS rate for patients in the high-risk group was lower than that for patients in the low-risk group $(96.9 \%$ vs. $84.2 \%$, $p<0.001$; Supplementary Fig. 6e).

As shown in Fig. 3, there were no significant differences in FFS between the two treatment models in the whole validation cohort and the low-risk group ( $p=0.1, p=0.52$ ). However, patients undergoing induction chemotherapy followed by concurrent chemoradiotherapy had a higher survival rate than those undergoing concurrent chemoradiotherapy alone in the high-risk group (3-year FFS: $92.2 \%$ vs. 80.2\%; HR: 0.34, 95\% CI: 0.13-0.93, $p=0.028$; Fig. 3f).

\section{Discussion}

In this large cohort study, $\left[{ }^{18} \mathrm{~F}\right] \mathrm{FDG}$ PET/CT was more accurate than MRI for detecting metastatic cervical lymph nodes, which provided firm evidence for precisely identifying T3N1M0 patients by both $\left[{ }^{18}\right.$ F]FDG PET/CT and MRI. Radiologic lymph node characteristics, including SUVmax$\mathrm{N}$ higher than 9.3, nodal necrosis, and grade 3 radiologic extranodal extension, were independent prognostic factors for nasopharyngeal carcinoma patients staged as T3N1M0. Accordingly, high-risk and low-risk groups could be stratified by these risk factors instead of sex and EBV DNA load. We demonstrated that only patients in the high-risk group could benefit from the addition of induction chemotherapy. These findings were verified again by the validation cohort from our clinical trial.

In this study, EBV DNA load was not confirmed for separating the subgroup of T3N1M0. As 206 and 96 patients in the training and validation cohorts had EBV DNA loads higher than 2000 copies $/ \mathrm{mL}$, an insufficient sample size may not justify this result. Similarly, female patients also did not show a superior survival rate. Consistent with a prior study
[29], $\left[{ }^{18}\right.$ F]FDG PET/CT did have better diagnostic accuracy than conventional imaging in nasopharyngeal carcinoma, as proven by the histological results of the lymph nodes. Therefore, the subgroup of T3N1M0 staged by both $\left[{ }^{18} \mathrm{~F}\right]$ FDG PET/CT and MRI in this study showed a relatively high survival rate, which was close to the reported rate of stage II patients [30]. As a result, extremely strong predictive markers are required for further separation of this subgroup.

$\left[{ }^{18} \mathrm{~F}\right] \mathrm{FDG} \mathrm{PET} / \mathrm{CT}$, as functional imaging, provides metabolic information and can guide prognostication [31]. Prior studies have reported that SUVmax-T, SUVmax-N, and $\mathrm{SUV}_{75 \%}$ of primary tumors are prognostic factors for nasopharyngeal carcinoma [32, 33]. Thus, it was not absurd that SUVmax-N also acted as an independent prognostic factor in the subgroup of patients with T3N1M0. Pathologic extranodal extension has been introduced into the $\mathrm{N}$ classification for nonviral-related head and neck cancer in the 8th edition of the AJCC/UICC staging system. Due to the radiotherapy-based primary treatment, pathologic extranodal extension is not available for nasopharyngeal carcinoma. However, radiologic extranodal extension based on MRI or $\mathrm{CT}$ has good specificity and sensitivity (ranging from 70 to $90 \%$ ) in predicting pathologic extranodal extension in head and neck cancer [34]. The specificity of radiologic extranodal extension infiltrating adjacent structures is nearly $100 \%$, consistent with pathologic extranodal extension [18, 35]. Therefore, radiologic extranodal extension based on MRI is an accepted surrogate of pathologic extranodal extension for nasopharyngeal carcinoma. Similar to previous studies [19-21], the most severe radiologic extranodal extension with the involvement of adjacent structures was correlated with poor survival outcomes in the subgroup of T3N1M0 nasopharyngeal carcinoma. In addition, nodal necrosis, a vital radiologic nodal feature, is a reliable sign for detecting nodal metastasis. MRI has similar sensitivity to CT in identifying nodal necrosis [27]. Previous studies indicated that nodal necrosis is a strong prognostic factor in nasopharyngeal carcinoma, as the survival rate of patients with nodal necrosis declined nearly $12 \%$ in comparison with that of patients without nodal necrosis [36]. Therefore, it was not unreasonable that nodal necrosis was a significant predictor of outcomes in the T3N1M0 subgroup.

In previous studies [19-21], the TNM stage of enrolled patients varied from stage I to stage IVa, which contained significant heterogeneity. The confounding factors, including T stage, nodal size, nodal level, nodal laterality, and treatment modes, could not be completely eliminated. In our study, the metastatic lymph nodes of T3N1M0 patients were located in the unilateral upper cervical region, which fully controlled the covariate factors of $\mathrm{T}$ stage and radiological lymph node characteristics, such as nodal level and nodal laterality. As mentioned above, after eliminating confounding factors, our study confirmed that the three radiological 
lymph node characteristics, namely SUVmax-N, extranodal extension, and nodal necrosis, were closely related to survival outcome, especially distant metastasis. Perhaps if lymph nodes have a high metabolic rate and tumors spread outside the nodal capsule, tumor cells can easily enter the blood circulation and finally develop metastasis in distant organs. Although intensity-modulated radiotherapy delivers radical doses to the primary tumor and metastatic lymph nodes and can achieve excellent locoregional control [4], the high risk of distant metastasis for patients with these sorts of nodal characteristics cannot be reduced. As we found in the present study, high-risk T3N1M0 patients had a similar 5-year FFS rate to patients who were enrolled in clinical trials of induction chemotherapy [37]. Given the confirmed benefit of induction chemotherapy in locoregionally advanced nasopharyngeal carcinoma from randomized controlled trials [5-7] and meta-analyses [38, 39], it is highly reasonable that induction chemotherapy plus concurrent chemoradiotherapy can improve the survival rate of highrisk T3N1M0 patients.

There are several advantages of this study. First, all patients were restaged by $\left[{ }^{18} \mathrm{~F}\right] \mathrm{FDG} \mathrm{PET} / \mathrm{CT}$ and MRI according to the 8th AJCC/UICC staging system. The subset of patients who underwent biopsy of certain cervical lymph nodes demonstrated the accuracy of $\left[{ }^{18} \mathrm{~F}\right]$ FDG PET/ $\mathrm{CT}$ in detecting positive lymph nodes, which supported the reliability of the T3N1M0 staging of the patients. Notably, all eligible patients were upper cervical lymph node positive and unilateral lymph node positive, which fully eliminated covariate factors, including $\mathrm{T}$ stage, nodal laterality, nodal level, and nodal size. In addition, the sample size was relatively large, and the results were verified by a validation cohort. Limitations of this study should also be noted. First, this was a single-center study, and WHO type III was the predominant pathology type. Second, the follow-up duration of the validation cohort was not long enough. Hence, subsequent follow-up is warranted.

\section{Conclusion}

In conclusion, T3N1M0 patients could be diagnosed more accurately by both $\left[{ }^{18} \mathrm{~F}\right] \mathrm{FDG}$ PET/CT and MRI. The radiologic score of lymph node characteristics based on MRI and $\left[{ }^{18} \mathrm{~F}\right]$ FDG PET/CT could correctly identify high-risk patients who can obtain additional survival benefit from induction chemotherapy and it could be used to guide individualized treatment for nasopharyngeal carcinoma patients staged with T3N1M0 in clinical practice.

Supplementary Information The online version contains supplementary material available at https://doi.org/10.1007/s00330-021-08460-1.
Acknowledgements We thank Yun He, MD, Department of Radiology, Sun Yat-sen University Cancer Center, China, for helping us evaluate the inconsistent MR images.

Funding This work was supported by the Sun Yat-sen University Clinical Research 5010 Program (2015020), the Sci-Tech Project Foundation of Guangdong Province (2016A020215087), the Natural Science Foundation of Guangdong Province (2019A1515010300), Medical Scientific Research Foundation of Guangdong Province, China (A2016197), and Scientific research project of Guangdong Bureau of traditional Chinese Medicine (20201069).

\section{Declarations}

Guarantor The scientific guarantor of this publication is Fang-Yun Xie.

Conflict of interest The authors of this manuscript declare no relationships with any companies whose products or services may be related to the subject matter of the article.

Statistics and biometry No complex statistical methods were necessary for this paper.

Informed consent Written informed consent was obtained from all subjects (patients) in this study.

Ethical approval Institutional Review Board approval was obtained.

\section{Methodology}

- retrospective plus prospective validation

- observational

- performed at one institution

Open Access This article is licensed under a Creative Commons Attribution 4.0 International License, which permits use, sharing, adaptation, distribution and reproduction in any medium or format, as long as you give appropriate credit to the original author(s) and the source, provide a link to the Creative Commons licence, and indicate if changes were made. The images or other third party material in this article are included in the article's Creative Commons licence, unless indicated otherwise in a credit line to the material. If material is not included in the article's Creative Commons licence and your intended use is not permitted by statutory regulation or exceeds the permitted use, you will need to obtain permission directly from the copyright holder. To view a copy of this licence, visit http://creativecommons.org/licenses/by/4.0/.

\section{References}

1. Sung H, Ferlay J, Siegel RL et al (2021) Global cancer statistics 2020: GLOBOCAN estimates of incidence and mortality worldwide for 36 cancers in 185 countries. CA Cancer J Clin. https:// doi.org/10.3322/caac. 21660

2. Chen Y-P, Chan ATC, Le Q-T, Blanchard P, Sun Y, Ma J (2019) Nasopharyngeal carcinoma. Lancet 394:64-80

3. Pan JJ, Ng WT, Zong JF et al (2016) Prognostic nomogram for refining the prognostication of the proposed 8th edition of the AJCC/UICC staging system for nasopharyngeal cancer in the era of intensity-modulated radiotherapy. Cancer 122:3307-3315

4. Sun X, Su S, Chen C et al (2014) Long-term outcomes of intensity-modulated radiotherapy for 868 patients with nasopharyngeal carcinoma: an analysis of survival and treatment toxicities. Radiother Oncol 110:398-403 
5. Cao SM, Yang Q, Guo L et al (2017) Neoadjuvant chemotherapy followed by concurrent chemoradiotherapy versus concurrent chemoradiotherapy alone in locoregionally advanced nasopharyngeal carcinoma: a phase III multicentre randomised controlled trial. Eur J Cancer 75:14-23

6. Sun Y, Li W-F, Chen N-Y et al (2016) Induction chemotherapy plus concurrent chemoradiotherapy versus concurrent chemoradiotherapy alone in locoregionally advanced nasopharyngeal carcinoma: a phase 3, multicentre, randomised controlled trial. Lancet Oncol 17:1509-1520

7. Zhang Y, Chen L, Hu GQ et al (2019) Gemcitabine and cisplatin induction chemotherapy in nasopharyngeal carcinoma. N Engl $\mathrm{J}$ Med 381:1124-1135

8. DG P SS, (2021) Head and Neck Cancers, Version 3.2021, NCCN Clinical Practice Guidelines in Oncology. J Natl Compr Canc Netw 18:873-898

9. Lan XW, Xiao Y, Zou XB, Zhang XM, OuYang PY, Xie FY (2017) Outcomes of adding induction chemotherapy to concurrent chemoradiotherapy for stage T3N0-1 nasopharyngeal carcinoma: a propensity-matched study. Onco Targets Ther 10:3853-3860

10. Xu C, Zhang S, Li WF et al (2019) Selection and validation of induction chemotherapy beneficiaries among patients with T3N0, T3N1, T4N0 nasopharyngeal carcinoma using Epstein-Barr virus DNA: a joint analysis of real-world and clinical trial data. Front Oncol 9:1343

11. Tang LQ, Chen QY, Fan W et al (2013) Prospective study of tailoring whole-body dual-modality $[18 \mathrm{~F}]$ fluorodeoxyglucose positron emission tomography/computed tomography with plasma Epstein-Barr virus DNA for detecting distant metastasis in endemic nasopharyngeal carcinoma at initial staging. J Clin Oncol 31:2861-2869

12. Cho H, Kim SH, Kim H et al (2018) Lymph node with the highest FDG uptake predicts distant metastasis-free survival in patients with locally advanced nasopharyngeal carcinoma. Clin Nucl Med 43:e220-e225

13. Xie P, Yue JB, Fu Z, Feng R, Yu JM (2010) Prognostic value of $18 \mathrm{~F}-\mathrm{FDG}$ PET/CT before and after radiotherapy for locally advanced nasopharyngeal carcinoma. Ann Oncol 21:1078-1082

14. Hung TM, Fan KH, Kang CJ et al (2020) Lymph node-to-primary tumor standardized uptake value ratio on PET predicts distant metastasis in nasopharyngeal carcinoma. Oral Oncol 110:104756

15. Lin P, Min M, Lee M et al (2016) Prognostic utility of (18)F-FDG PET-CT performed prior to and during primary radiotherapy for nasopharyngeal carcinoma: index node is a useful prognostic imaging biomarker site. Radiother Oncol 120:87-91

16. Li WF, Sun Y, Mao YP et al (2013) Proposed lymph node staging system using the International Consensus Guidelines for lymph node levels is predictive for nasopharyngeal carcinoma patients from endemic areas treated with intensity modulated radiation therapy. Int J Radiat Oncol Biol Phys 86:249-256

17. Guo Q, Pan J, Zong J et al (2015) Suggestions for lymph node classification of UICC/AJCC staging system: a retrospective study based on 1197 nasopharyngeal carcinoma patients treated with intensity-modulated radiation therapy. Medicine (Baltimore) 94:e808

18. Prabhu RS, Magliocca KR, Hanasoge S et al (2014) Accuracy of computed tomography for predicting pathologic nodal extracapsular extension in patients with head-and-neck cancer undergoing initial surgical resection. Int J Radiat Oncol Biol Phys $88: 122-129$

19. Mao Y, Wang S, Lydiatt W et al (2021) Unambiguous advanced radiologic extranodal extension determined by MRI predicts worse outcomes in nasopharyngeal carcinoma: potential improvement for future editions of $\mathrm{N}$ category systems. Radiother Oncol 157:114-121

20. Ai QY, King AD, Poon DMC et al (2019) Extranodal extension is a criterion for poor outcome in patients with metastatic nodes from cancer of the nasopharynx. Oral Oncol 88:124-130

21. Lu T, Hu Y, Xiao Y et al (2019) Prognostic value of radiologic extranodal extension and its potential role in future $\mathrm{N}$ classification for nasopharyngeal carcinoma. Oral Oncol 99:104438

22. Hu Y, Lu T, Huang SH et al (2019) High-grade radiologic extranodal extension predicts distant metastasis in stage II nasopharyngeal carcinoma. Head Neck 41:3317-3327

23. Ng SH, Chan SC, Yen TC et al (2009) Staging of untreated nasopharyngeal carcinoma with PET/CT: comparison with conventional imaging work-up. Eur J Nucl Med Mol Imaging 36:12-22

24. Xiao BB, Chen QY, Sun XS et al (2021) Low value of wholebody dual-modality [18f]fluorodeoxyglucose positron emission tomography/computed tomography in primary staging of stage I-II nasopharyngeal carcinoma: a nest case-control study. Eur Radiol 31:5222-5233

25. Amin MB (2017) American Joint Committee on Cancer. AJCC cancer staging manual, 8th edn. Springer, New York

26. van den Brekel MW, Stel HV, Castelijns JA et al (1990) Cervical lymph node metastasis: assessment of radiologic criteria. Radiology 177:379-384

27. King AD, Tse GM, Ahuja AT et al (2004) Necrosis in metastatic neck nodes: diagnostic accuracy of CT, MR imaging, and US. Radiology 230:720-726

28. Newcombe RG (1998) Two-sided confidence intervals for the single proportion: comparison of seven methods. Stat Med 17:857-872

29. Gordin A, Golz A, Daitzchman M et al (2007) Fluorine-18 fluorodeoxyglucose positron emission tomography/computed tomography imaging in patients with carcinoma of the nasopharynx: diagnostic accuracy and impact on clinical management. Int $\mathrm{J}$ Radiat Oncol Biol Phys 68:370-376

30. Zhang LN, Gao YH, Lan XW et al (2015) Propensity score matching analysis of cisplatin-based concurrent chemotherapy in low risk nasopharyngeal carcinoma in the intensity-modulated radiotherapy era. Oncotarget 6:44019-44029

31. Peng H, Dong D, Fang MJ et al (2019) Prognostic value of deep learning PET/CT-based radiomics: potential role for future individual induction chemotherapy in advanced nasopharyngeal carcinoma. Clin Cancer Res 25:4271-4279

32. Hung TM, Wang HM, Kang CJ et al (2013) Pretreatment (18) F-FDG PET standardized uptake value of primary tumor and neck lymph nodes as a predictor of distant metastasis for patients with nasopharyngeal carcinoma. Oral Oncol 49:169-174

33. Hsieh TC, Hsieh CY, Yang TY et al (2015) [18F]-Fluorodeoxyglucose positron emission tomography standardized uptake value as a predictor of adjuvant chemotherapy benefits in patients with nasopharyngeal carcinoma. Oncologist 20:539-545

34. Su Z, Duan Z, Pan W et al (2016) Predicting extracapsular spread of head and neck cancers using different imaging techniques: a systematic review and meta-analysis. Int J Oral Maxillofac Surg 45:413-421

35. Almulla A, Noel CW, Lu L et al (2018) Radiologic-pathologic correlation of extranodal extension in patients with squamous cell carcinoma of the oral cavity: implications for future editions of the TNM classification. Int J Radiat Oncol Biol Phys 102:698-708

36. Lan M, Huang Y, Chen CY et al (2015) Prognostic value of cervical nodal necrosis in nasopharyngeal carcinoma: analysis of 1800 patients with positive cervical nodal metastasis at MR imaging. Radiology 276:536-544 
37. Li WF, Chen NY, Zhang N et al (2019) Concurrent chemoradiotherapy with/without induction chemotherapy in locoregionally advanced nasopharyngeal carcinoma: long-term results of phase 3 randomized controlled trial. Int J Cancer 145:295-305

38. OuYang PY, Zhang XM, Qiu XS et al (2019) A pairwise metaanalysis of induction chemotherapy in nasopharyngeal carcinoma. Oncologist 24:505-512
39. Yang SS, Guo JG, Liu JN et al (2020) Effect of induction chemotherapy in nasopharyngeal carcinoma: an updated meta-analysis. Front Oncol 10:591205

Publisher's note Springer Nature remains neutral with regard to jurisdictional claims in published maps and institutional affiliations. 